Research Paper

\title{
Clinicopathologic Features and Prognostic Implications of Golgi Phosphoprotein 3 in Non-small Cell Lung Cancer: A Meta-analysis
}

\author{
Wenhua Shi, Wei Feng, Jian Wang, Cui Zhai, Qianqian Zhang, Qingting Wang, Yang Song, Xin Yan, Limin \\ Chai, Pengtao Liu, Yuqian Chen, Cong Li, Manxiang Li ${ }^{\bowtie}$ \\ Department of Respiratory and Critical Care Medicine, The First Affiliated Hospital of Xi'an Jiaotong University, Xi'an, Shaanxi 710061, China \\ $\triangle$ Corresponding author: Dr. Manxiang Li; Department of Respiratory and Critical Care Medicine, the First Affiliated Hospital of Xi'an Jiaotong University; No. \\ 277, West Yanta Road, Xi' an, Shaanxi 710061, China; Telephone: +86-029-85324053; Fax: +86-029-85324053; E-mail address: manxiangli@hotmail.com. \\ (c) The author(s). This is an open access article distributed under the terms of the Creative Commons Attribution License (https://creativecommons.org/licenses/by/4.0/). \\ See http://ivyspring.com/terms for full terms and conditions.
}

Received: 2018.09.19; Accepted: 2019.04.11; Published: 2019.10.04

\begin{abstract}
Background: A number of studies have investigated the role of Golgi phosphoprotein-3 (GOLPH3) in the pathogenesis and progression of non-small cell lung cancer (NSCLC). However, the results of previous studies are heterogeneous and controversial. The aim of this meta-analysis was to clarify its association with the clinicopathological characteristics of patients and evaluate the prognostic significance of GOLPH3 in NSCLC.

Methods: A systematic search was conducted through PMC, PubMed, Medline, Web of Science, Chinese National Knowledge Infrastructure and Wanfang database. The odds ratio (OR) and hazard ratio (HR) with $95 \% \mathrm{Cl}$ were calculated by STATA 12.0 .

Results: 8 qualified studies with a total of 1001 patients with NSCLC were included. Pooled results showed that GOLPH3 was highly expressed in tumor tissues compared with adjacent lung tissues (OR, 7.55), and overexpression of GOLPH3 was significantly correlated with advanced clinical stage (OR, 3.42), poor differentiation of tumor (OR, 1.97) and positive lymph node metastasis (OR, 2.58), but no association with histological type, gender, age or tumor size was found in NSCLC patients. In addition, the pooled HR for overall survival was 1.79 by univariate analysis and 1.91 by multivariate analysis. The pooled HR for progression-free survival was 2.50 .

Conclusions: GOLPH3 could be a risk factor for progression of NSCLC and might act as a potential prognostic biomarker for NSCLC patients.
\end{abstract}

Key words: GOLPH3; non-small cell lung cancer; biomarker; prognosis; meta-analysis

\section{Introduction}

Lung cancer is the most common malignancy and the leading cause of cancer-related death worldwide, with 234,030 new cases and 154,050 deaths estimated for the year 2018 in United States [1]. Non-small-cell lung carcinoma (NSCLC) is the main pathological type of lung cancer, accounting for more than $80 \%$ of the cases [2]. Clinically, the majority of NSCLC patients are often diagnosed at an advanced stage and ineligible for surgical resection with a curative intent [3], making successful treatment more difficult and overall survival poor despite the development of the molecular targeted agents [4] and the therapeutic strategy [5]. It has been demonstrated that the decreased survival rate is partially determined by the subtleness of symptoms, delayed diagnosis, concomitant diseases and limited therapeutic options [6, 7]. In recent years, a multitude of potentially useful biomarkers have emerged and established important applications in early diagnosis, risk stratification and prognosis assessment of lung 
cancer, which is helpful to implement timely intervention and improve the clinical outcome of patients.

Golgi phosphoprotein 3 (GOLPH3), a phosphorylated protein with a molecular weight of 34 $\mathrm{kDa}$, is involved in anterograde and retrograde Golgi trafficking [8-10]. Previous studies have demonstrated that GOLPH3 is highly expressed in various types of human cancer, including NSCLC [11], gastric cancer [12], breast cancer [13], hepatocellular cancer [14] and prostate cancer [15], and overexpression of GOLPH3 promotes cancer cell proliferation, migration and invasion[15, 16]. In addition, GOLPH3 gene expression has been shown to be associated with poor prognosis and chemotherapy resistance in patients with cancer $[10,17]$. Although quite a number of studies have investigated the clinical role of GOLPH3 in NSCLC and indicated that GOLPH3 could be a risk factor and prognostic biomarker for NSCLC, the relationships of GOLPH3 expression with clinicopathologic features in patients with NSCLC are inconsistent and controversial. Therefore, we performed a meta-analysis including all eligible studies to reveal the authentic value of GOLPH3 in cancer outcome and clinicopathological characteristics thereby providing more evidence for clinical practice.

\section{Methods}

\section{Search strategy}

Articles were identified using a comprehensive literature search in PMC, Pubmed, Medline, Web of Science, Chinese National Knowledge Infrastructure (CNKI) and Wanfang databases. The time scope that we defined was from databases inception to January 2019. The combination of the Medical Subject Headings (MeSH) and the keywords that we used for searching literature was: "lung cancer" or "lung tumor" or "lung carcinoma" or "NSCLC" or "non-small cell lung cancer") and ("GPP34 protein" or "Golgi phosphoprotein 3" or "GOLPH3"). The relevant reviews and the references of the included studies were also examined manually to find additional data.

\section{Inclusion and exclusion criteria of literature}

Studies included in this meta-analysis were required to meet the following criteria: (1) cancers were diagnosed histologically; (2) the expression of GOLPH3 was evaluated by immunohistochemistry; (3) clinical and histological data of NSCLC patients were provided; (4) carcinoma specimens were taken prior to chemotherapy, radiotherapy and other drug treatment; (5) articles had to be published in English or Chinese. The exclusion criteria: (1) studies that contained duplicated data; (2) editorials, letters, conference proceedings, comments and case reports; (3) studies without relevant data; (4) studies not performed in humans.

\section{Data extraction and quality assessment}

Two independent reviewers extracted all the available data from each eligible study. The following information were extracted: author, publication year, GOLPH3 assessment method, follow-up period, numbers of patients, case number of different groups, age of patients, tumor size, cut-off values for age and tumor size, histological classification, tumor differentiation degree, tumor node metastases, $\mathrm{T}$ descriptor, tumor-node-metastasis (TNM) classification, hazard ratio (HR) and corresponding 95\% confidence intervals (CI) for OS and PFS. The HRs (95 \% CIs) by univariate analysis and multivariate analysis were extracted directly from some studies. When HR and 95\% CI were not provided in the article, these values were estimated from the Kaplan-Meier curves using the method described previously $[18,19]$. Quality assessment of included studies was performed by two independent authors based on Newcastle-Ottawa Scale grading system [20]. Any disagreements were solved by discussion with a third team member.

\section{Statistical analysis}

We calculated the OR and 95\% CI to determine the association of GOLPH3 expression and clinicopathological characteristics in patients with NSCLC. If OR > 1 and the 95\% CI doesn't include 1, it indicates that there is an association between high GOLPH3 expression and clinicopathological variable. The impact of GOLPH3 overexpression on OS and PFS of NSCLC patients was estimated by HR and its 95\% CI and HR > 1 represents a poor prognosis. We performed this meta-analysis using two different approaches (fixed and random effects model analysis) based on heterogeneity. Potential heterogeneity among the studies was quantified using chi-squared test and $\mathrm{I}^{2}$ [21], which was defined statistical significance at a level of $\mathrm{p}<0.1$ or $\mathrm{I}^{2}>50 \%$. A fixed effects model was selected in the absence of significant heterogeneity. Otherwise, a random effects model was applied [22]. If necessary, we performed subgroup analysis to explore potential sources of heterogeneity. Egger's test was implemented to visually identify publication bias [23], and sensitivity analysis was conducted by eliminating each study to examine the robustness of the results. All statistical analyses were performed using STATA software (Version 12.0; STATA Corporation, College Station, 
TX), and a two-sided $p$ value less than 0.05 was considered statistically significant.

\section{Results}

\section{Searching of literature}

The diagram of the study selection process is shown in Figure 1. During the initial search of the medical literature related to GOLPH3 and lung cancer, we identified 291 studies. Among these articles, 65 duplicate studies and 183 irrelevant studies were excluded after reviewing title and abstracts of articles. Subsequently, 43 articles were excluded for the following reasons: reviews $(n=14)$, letters or conference abstracts $(n=2)$, cell research $(n=17)$, animal research $(n=2)$. Finally, 8 articles met the inclusion criteria and were included for further analysis. A manual search of the reference lists of the 8 studies did not yield any eligible studies.

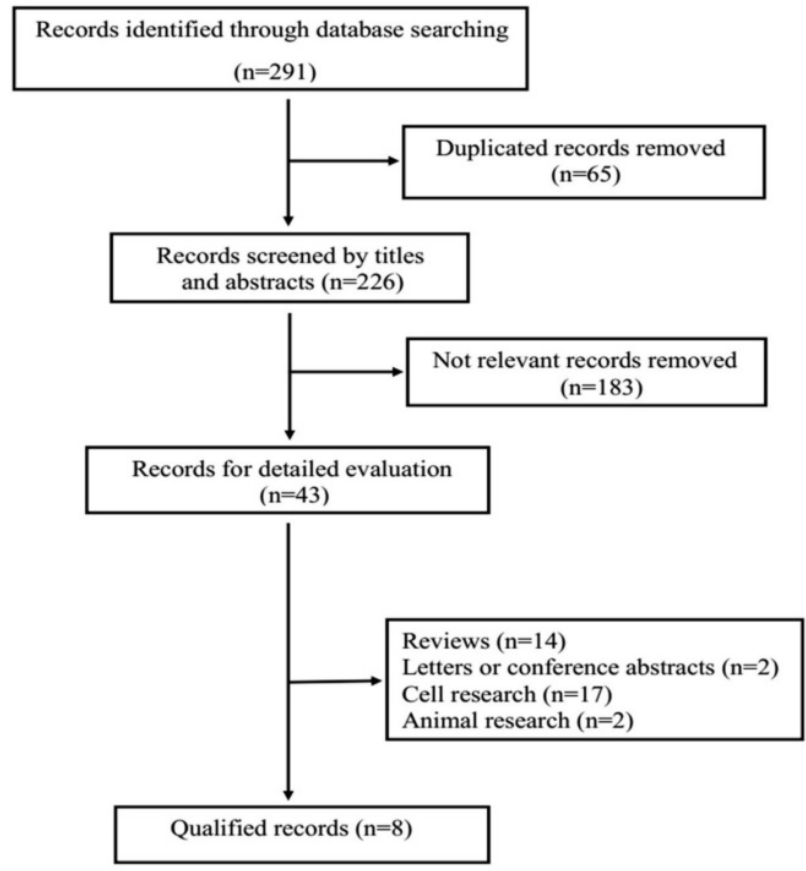

Figure 1. Flow chart of the selection for the meta-analysis.

\section{Characteristics of included studies}

As shown in Table 1, a total of eight studies [11, 16, 24-29] with 1001 newly diagnosed NSCLC patients were assessed for this analysis. All eligible studies were published from 2014 to 2018 in Asia, with a sample size ranging from 57 to 230, and the median follow-up of the studies was 33.9-52 months. All the studies were retrospective study design and $33.9-74.0 \%$ of the patients were male. The expression of GOLPH3 in cancer and adjacent normal tissues from NSCLC patients was detected by immunohistochemistry (IHC) staining, which included assignment of staining intensity and area of positive staining using the semi-quantitative scoring system. The histological classification of lung cancer mainly contained lung adenocarcinoma (518 patients) and lung squamous cell carcinoma (458 patients). The quality of included studies assessed by Newcastle-Ottawa scale was considered acceptable, as all the studies had more than 5 stars of scores (Table 1).

\section{GOLPH3 expression in cancer and adjacent normal lung tissues}

Six studies [16, 25-29] provided GOLPH3 expression data in 711 lung cancer tissue samples and 461 adjacent normal lung tissue samples (Table 2). The overall OR was 7.55 (95\% CI = 3.20-17.80, p < 0.001) via a random effects model analysis $\left(\mathrm{I}^{2}=83.8 \%\right.$, $\mathrm{p}<$ 0.001) (Figure 2A). After excluding the study by Ming Lu et al. [26] which was performed in patients with stage I NSCLC only, the heterogeneity decreased $\left(\mathrm{I}^{2}=67.8 \%\right.$, $\mathrm{p}=0.014)$ and the overall OR was $10.04(95 \% \mathrm{CI}=$ 4.86-20.73, $\mathrm{p}<0.001$ ) (Figure 2B). These results indicated that expression level of GOLPH3 was dramatically higher in the cancer tissues of NSCLC patients compared with adjacent lung tissues and the study by Ming Lu et al. might partly contribute to the heterogeneity.

\section{GOLPH3 expression and clinical stage}

Five studies $[16,24,25,27,28]$ reported relevant data concerning the expression of GOLPH3 in different clinical stages of NSCLC patients (Table 2). Our pooled result showed that the overall OR was $3.42(95 \% \mathrm{CI}=1.85-6.30, \mathrm{p}<0.001)$ via a random effects model analysis $\left(\mathrm{I}^{2}=55.2 \%, \mathrm{p}=0.063\right)$ (Figure 3A). Subgroup analysis stratified by sample size showed that the summary OR was $2.87(95 \% \mathrm{CI}=$ $\left.1.95-4.20, \mathrm{p}<0.001 ; \mathrm{I}^{2}=0 \%, \mathrm{p}=0.768\right)$ in studies with sample size $\geq 100$ and 61.33 (95\% CI $=7.19-523.22, p<$ 0.001 ) in studies with fewer subjects (Figure 3B). Significant association existed between high GOLPH3 expression and clinical stage of patients, indicating that expression level of GOLPH3 was dramatically higher in advanced stage than that in early stage.

\section{GOLPH3 expression and tumor differentiation}

Six studies [16, 24-27, 29], comprising of 765 NSCLC patients, reported data concerning the expression of GOLPH3 in different degree of tumor differentiation in NSCLC patients (Table 2). The overall OR was $1.97(95 \% \mathrm{CI}=1.34-2.88, \mathrm{p}=0.002)$ via a fixed effects model analysis $\left(\mathrm{I}^{2}=40.0 \%, \mathrm{p}=0.139\right)$ (Figure 4), suggesting that expression level of GOLPH3 was relatively higher in moderate and poor differentiation of tumor than that in well tumor differentiation. 
Table 1. Baseline characteristics of included studies.

\begin{tabular}{|c|c|c|c|c|c|c|c|c|c|c|c|}
\hline Authors & $\begin{array}{l}\text { Publication } \\
\text { year }\end{array}$ & Country & $\begin{array}{l}\text { Enrollment } \\
\text { period }\end{array}$ & Research design & $\begin{array}{l}\text { Resources of } \\
\text { samples }\end{array}$ & Test method & $\begin{array}{l}\text { Median } \\
\text { follow-up }\end{array}$ & $\begin{array}{l}\text { No. of } \\
\text { cases }\end{array}$ & Male (\%) & $\begin{array}{l}\text { Clinical stage of } \\
\text { patients }\end{array}$ & score \\
\hline SC Tang & 2018 & China & $2011-2016$ & Retrospective & Tumor tissue & Immunohistochemistry & - & 60 & $71.67 \%$ & - & 7 \\
\hline WF Tang & 2016 & China & 2009-2013 & Retrospective & Tumor tissue & Immunohistochemistry & 35 months & 100 & $74.00 \%$ & stage I- III & 6 \\
\hline R Wang & 2015 & China & 2002-2012 & Retrospective & Tumor tissue & Immunohistochemistry & - & 230 & $70.87 \%$ & stage I- IV & 5 \\
\hline QY Wei & 2015 & China & 2001-2005 & Retrospective & Tumor tissue & Immunohistochemistry & - & 57 & $70.18 \%$ & stage I- IV & 6 \\
\hline $\mathrm{M} \mathrm{Lu}$ & 2014 & China & 2000-2005 & Retrospective & Tumor tissue & Immunohistochemistry & 52 months & 116 & $58.62 \%$ & stage I & 6 \\
\hline Y Zhang & 2014 & China & 2001-2007 & Retrospective & Tumor tissue & Immunohistochemistry & 37 months & 145 & $52.41 \%$ & stage I- III & 7 \\
\hline JN Tang & 2014 & China & 2005-2012 & Retrospective & Tumor tissue & Immunohistochemistry & 33.9 months & 177 & $33.90 \%$ & stage I- III & 8 \\
\hline XL Lv & 2014 & China & 2004-2006 & Retrospective & Tumor tissue & Immunohistochemistry & 49 months & 116 & $62.07 \%$ & - & 7 \\
\hline
\end{tabular}

Table 2. Prevalence of high expression GOLPH3 staining in cancer tissues and adjacent normal tissues and according clinicopathological characteristics of patients with lung cancer.

\begin{tabular}{|c|c|c|c|c|c|c|c|c|c|c|c|c|c|c|c|c|c|c|c|}
\hline \multicolumn{20}{|c|}{ Expression of GOLPH3 (positive /all) (N) } \\
\hline \multirow[t]{2}{*}{ Author } & \multirow{2}{*}{$\begin{array}{l}\text { Adjacen } \\
\text { normal } \\
\text { tissues }\end{array}$} & \multirow{2}{*}{$\begin{array}{l}\text { Cancer } \\
\text { tissues }\end{array}$} & \multicolumn{2}{|c|}{ Gender } & \multicolumn{3}{|c|}{ Age (years) } & \multicolumn{2}{|c|}{ Tumor stage } & \multicolumn{2}{|c|}{$\begin{array}{l}\text { Tumor } \\
\text { differentiation }\end{array}$} & $\begin{array}{l}\text { Lymphatic } \\
\text { metastasis }\end{array}$ & \multicolumn{2}{|l|}{ Pathological type } & \multicolumn{5}{|c|}{ Tumor size } \\
\hline & & & $\overline{\text { Male }}$ & Female & $\begin{array}{l}< \\
\text { cut-off }\end{array}$ & $\begin{array}{l}\geq \\
\text { cut-off }\end{array}$ & $\begin{array}{l}\text { cut-off } \\
\text { f value }\end{array}$ & f I-II & III-IV & Well & $\begin{array}{l}\text { Moderate } \\
\text { to poor }\end{array}$ & Yes No & Adenocarcinoma & $\begin{array}{l}\text { Squamous cell } \\
\text { carcinoma }\end{array}$ & $\begin{array}{l}< \\
\text { cut-off }\end{array}$ & $\begin{array}{l}\geq \\
\text { cut-off }\end{array}$ & $\begin{array}{l}\text { cut-off } \\
\text { value }\end{array}$ & $\mathrm{T}_{1}$ & $\mathrm{~T}_{2}$ \\
\hline \multicolumn{2}{|c|}{ SC Tang - } & $28 / 60$ & $22 / 43$ & $6 / 17$ & $12 / 32$ & $16 / 28$ & 60 & - & - & - & - & $25 / 433 / 17$ & $14 / 30$ & $15 / 30$ & $8 / 20$ & $20 / 40$ & $5 \mathrm{~cm}$ & - & - \\
\hline $\begin{array}{l}\text { WF } \\
\text { Tang }\end{array}$ & $0 / 20$ & $52 / 100$ & $32 / 74$ & $20 / 26$ & $22 / 39$ & $30 / 61$ & 60 & $42 / 84$ & $10 / 16$ & $1 / 18$ & $51 / 82$ & $20 / 2932 / 71$ & $35 / 50$ & $17 / 50$ & $34 / 61$ & $18 / 39$ & $5 \mathrm{~cm}$ & - & - \\
\hline R Wang & & 230 & $102 / 163$ & $42 / 67$ & $96 / 145$ & $548 / 85$ & 65 & $89 / 160$ & $55 / 70$ & $5 / 12$ & $139 / 218$ & $54 / 85$ 90/145 & $588 / 134$ & $54 / 90$ & - & - & - & $45 / 8$ & $8663 / 98$ \\
\hline QY Wei & $1 / 19$ & $33 / 57$ & $21 / 40$ & $12 / 17$ & $26 / 41$ & $7 / 16$ & 65 & $9 / 32$ & $24 / 25$ & $18 / 32$ & $15 / 25$ & $28 / 345 / 23$ & $10 / 23$ & $23 / 34$ & $6 / 11$ & $27 / 46$ & $3 \mathrm{~cm}$ & - & - \\
\hline M Lu & $24 / 57$ & $68 / 116$ & $42 / 68$ & $26 / 48$ & $29 / 58$ & $39 / 58$ & 65 & - & - & $17 / 31$ & $51 / 85$ & - & $35 / 66$ & $33 / 50$ & - & - & - & $24 / 4$ & $4644 / 70$ \\
\hline Y Zhang & $33 / 145$ & $104 / 145$ & $54 / 76$ & $50 / 69$ & $69 / 94$ & $35 / 51$ & 65 & $57 / 90$ & $47 / 55$ & $22 / 40$ & $82 / 105$ & $59 / 7245 / 73$ & $46 / 61$ & $48 / 65$ & $21 / 31$ & $83 / 114$ & $43 \mathrm{~cm}$ & - & - \\
\hline JN Tang & $18 / 177$ & $116 / 177$ & $36 / 60$ & $81 / 117$ & $56 / 86$ & $61 / 91$ & 60 & $86 / 122$ & $31 / 55$ & & - & $47 / 6170 / 116$ & $645 / 88$ & $72 / 89$ & $44 / 86$ & $73 / 91$ & $3 \mathrm{~cm}$ & - & - \\
\hline XL LV & $12 / 43$ & $67 / 116$ & $44 / 72$ & $23 / 44$ & $28 / 52$ & $39 / 64$ & 65 & - & - & $19 / 35$ & $48 / 81$ & $18 / 3049 / 86$ & $34 / 66$ & $33 / 50$ & - & - & - & $30 / 5$ & $5437 / 62$ \\
\hline
\end{tabular}

\section{GOLPH3 expression and histologic type}

Eight studies investigated the relationship between GOLPH3 expression and histologic type of lung cancer $[11,16,24-29]$. The histological classification in these studies mainly contained lung adenocarcinoma (518 patients) and lung squamous cell carcinoma (458 patients) (Table 2). The overall OR was $1.25(95 \% \mathrm{CI}=0.68-2.32, \mathrm{p}=0.474)$ via a random effects model analysis $\left(\mathrm{I}^{2}=79.9 \%, \mathrm{p}<0.001\right)$ (Figure 5A). Subgroup analysis stratified by sample size showed that the OR in studies with sample size $\geq 100$ was $1.14\left(95 \% \mathrm{CI}=0.54-2.42, \mathrm{p}=0.733 ; \mathrm{I}^{2}=84.7 \%, \mathrm{p}<\right.$ 0.001 ). And the OR in smaller studies (sample size < 100) was $1.72\left(95 \% \mathrm{CI}=0.74-4.01, \mathrm{p}=0.211 ; \mathrm{I}^{2}=22.9 \%\right.$, $\mathrm{p}=0.255$ ) (Figure 5B). No significant association existed, indicating that GOLPH3 expression was not related to histologic type of lung cancer.

\section{GOLPH3 expression and lymphatic metastasis}

Seven studies $[11,16,24,25,27-29]$ revealed the correlation between the expression level of GOLPH3 and different status of lymph node metastasis in patients with NSCLC, including 354 cases with lymphatic metastasis and 531 cases without lymphatic metastasis (Table 2). A random effects model was used due to the high heterogeneity $\left(\mathrm{I}^{2}=71.0 \%, \mathrm{p}=\right.$ $0.002)$ and the overall OR was 2.58 (95\% CI $=1.42-4.71$, $\mathrm{p}=0.001$ ) (Figure 6A). Subgroup analysis stratified by sample size showed that the summary OR in studies with more than 100 subjects was $1.68(95 \% \mathrm{CI}=$
1.21-2.32, $\left.\mathrm{p}<0.001 ; \mathrm{I}^{2}=41.5 \%, \mathrm{p}=0.145\right)$. And the OR for studies with fewer subjects (sample size $<100$ ) was $10.66\left(95 \% \mathrm{CI}=4.09-27.79, \mathrm{p}<0.001 ; \mathrm{I}^{2}=0.00 \%\right.$, p $=0.331$ ) (Figure 6B). These results suggested that the expression level of GOLPH3 was markedly higher in lymphatic metastasis group compared to non-lymphatic metastasis group.

\section{Association between GOLPH3 expression and gender, age and tumor size}

A total of 8 studies [11, 16, 24-29] assessed the relationship between GOLPH3 expression and general characteristics of patients (gender, age) (Table 2 ). The $I^{2}$ and $p$-value for heterogeneity were $53.6 \%$ and 0.035 , respectively. Therefore, the random effects model was used in gender analysis. Our results showed that increased GOLPH3 expression was not associated with gender of patients $(\mathrm{OR}=1.19,95 \% \mathrm{CI}$ $=0.78-1.80, p=0.42)$. Similar results were also found in the subgroup analyses according to sample size (n $\geq 100$ or $n<100$ ) (Table 3). Considering the use of different cut-off values for age ( 60 years in three studies [11, 16, 28] and 65 years in five studies [24-27, 29]), we performed separate meta-analyses and found that the overall OR in studies using 60 years and 65 years as age cut-off was $1.13(95 \% \mathrm{CI}=0.67-1.91, \mathrm{p}=$ $0.658)$ and $0.96(95 \% \mathrm{CI}=0.59-1.56, \mathrm{p}=0.854)$, respectively (Table 3 ). These results indicated that there was no significant association between GOLPH3 expression and age of patients. In addition, eight studies [11, 16, 24-29] revealed the correlation 
between the GOLPH3 expression and tumor size. Among these studies, three studies [25, 27, 28] used $3 \mathrm{~cm}$ as tumor size cut-off, two studies $[11,16]$ used a cut-off value of $5 \mathrm{~cm}$ and others [24, 26, 29] used the $\mathrm{T}$ descriptor from the TNM staging system. The results showed that no significant association between high expression of GOLPH3 and tumor size was found in three subgroups. Pooled ORs and heterogeneity test results for different subgroups in tumor size analysis are available in Table 3.

\section{Prognosis value of GOLPH3 in malignant tumors}

Seven studies [11, 16, 24, 26-29] with 856 NSCLC patients were eligible to evaluate the correlation between overexpression of GOLPH3 and OS of cancer patients, including seven studies [11,16, 24, 26-29] for univariate analysis and four studies [11, 16, 26, 28] for multivariate analysis (Table 4). The pooled HR was $1.79\left(95 \% \mathrm{CI}=1.47-2.17, \mathrm{p}<0.001 ; \mathrm{I}^{2}=14.3 \%, \mathrm{p}=\right.$ $0.321)$ for univariate analysis and $1.91(95 \% \mathrm{CI}=$ $\left.1.50-2.43, \mathrm{p}<0.001 ; \mathrm{I}^{2}=10.9 \%, \mathrm{p}=0.338\right)$ for multivariate analysis (Figure 7A and 7B). Considering the different method of obtaining HRs with $95 \% \mathrm{CI}$ for OS in univariate analysis (HRs and 95\% CI were extracted directly from three studies [11, 16, 28] and estimated by Kaplan-Meier survival curves in four studies [24, 26, 27, 29]), we further performed a subgroup analysis based on the method of obtaining HRs. The results showed that significant relationships between high expression of GOLPH3 and poor OS were exhibited in both subgroups ( $\mathrm{HR}=$ $1.83,95 \% \mathrm{CI}=1.44-2.34 ; \mathrm{HR}=1.71,95 \% \mathrm{CI}=1.24-2.35$, respectively) (Figure 7C). In addition, two studies [25, 26] including 261 NSCLC patients reported the HRs with $95 \% \mathrm{CI}$ for progression-free survival (PFS) in the multivariate models (Table 4). The pooled HR was $2.50(95 \% \mathrm{CI}=1.30-4.81, \mathrm{p}=0.006)$ using the random effects model (I2=54.7\%, $\mathrm{p}=0.137$ ) (Figure 7D). These results indicated that high GOLPH3 expression could predict a poor prognosis for NSCLC patients.

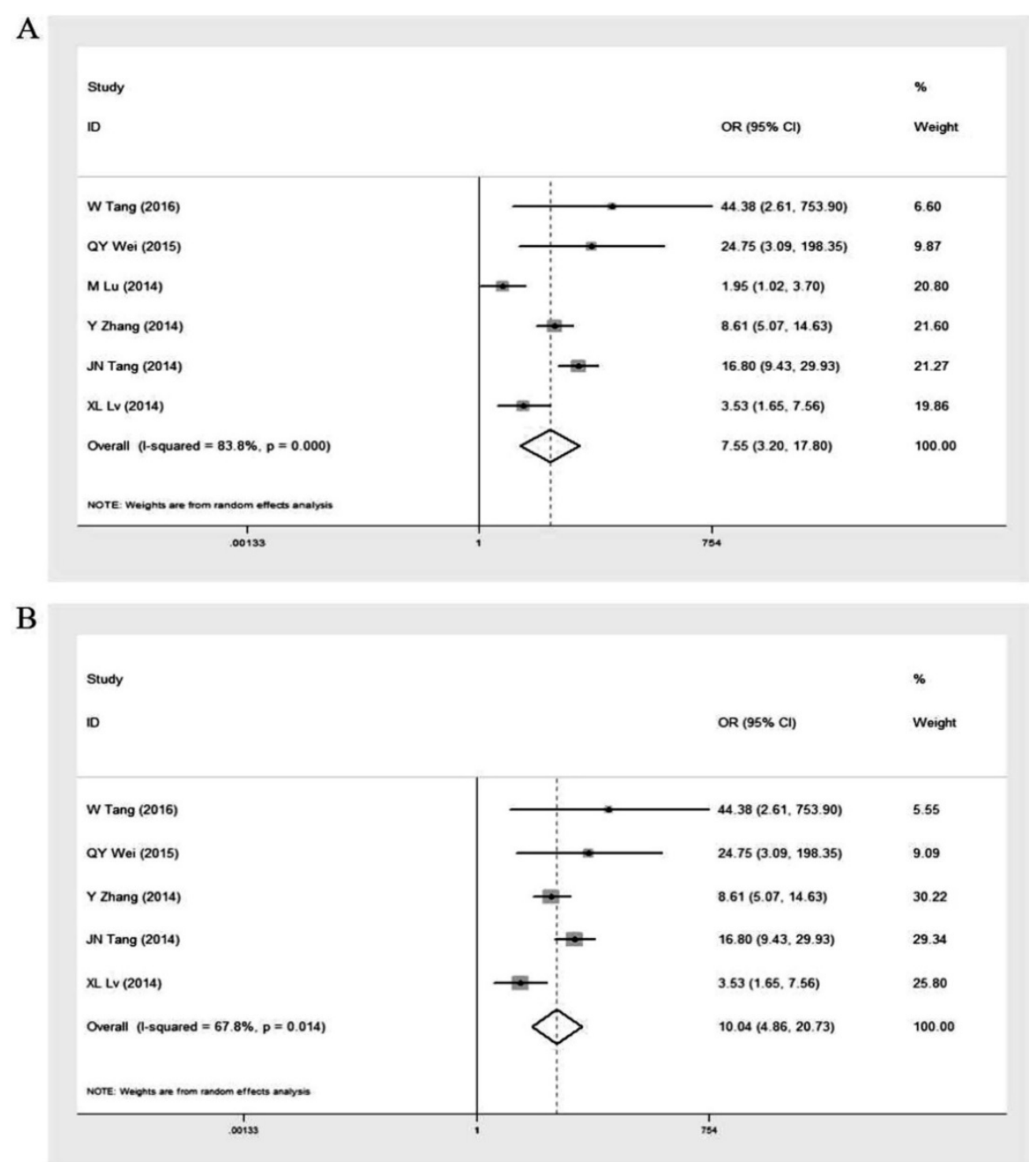

Figure 2. Forest plot of odds ratio (OR) for GOLPH3 expression in lung cancer tissue samples and adjacent normal lung tissue samples. A. including the study by $M$ Lu et al.; B. excluding the study by M Lu et al. cancer tissue vs adjacent normal tissue. If OR > 1 and the $95 \% \mathrm{Cl}$ doesn't include 1 , it indicates that expression level of GOLPH3 was dramatically higher in the cancer tissues of NSCLC patients compared with adjacent lung tissues. 
A

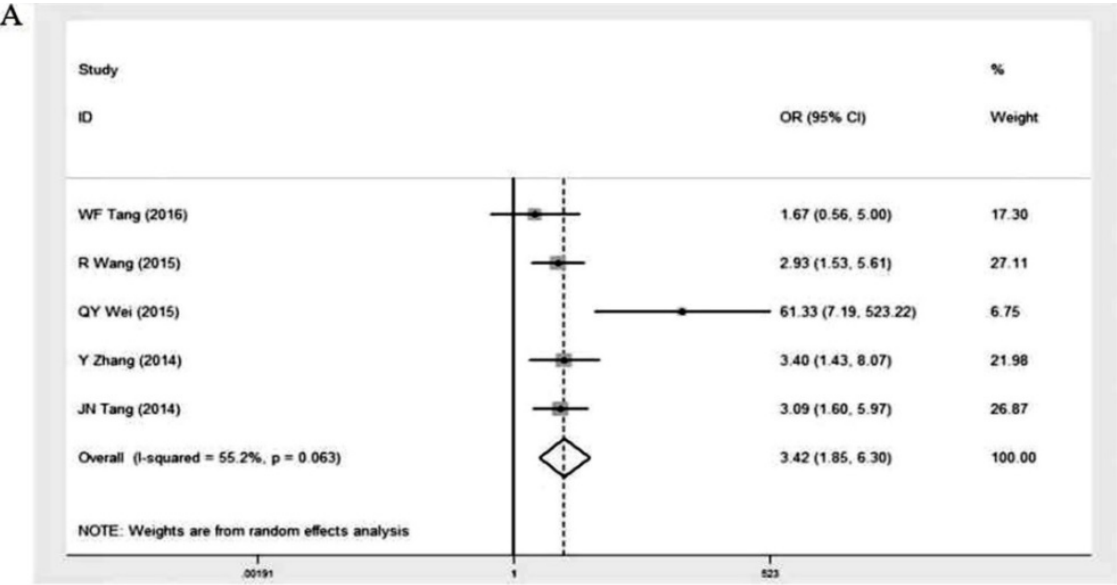

B

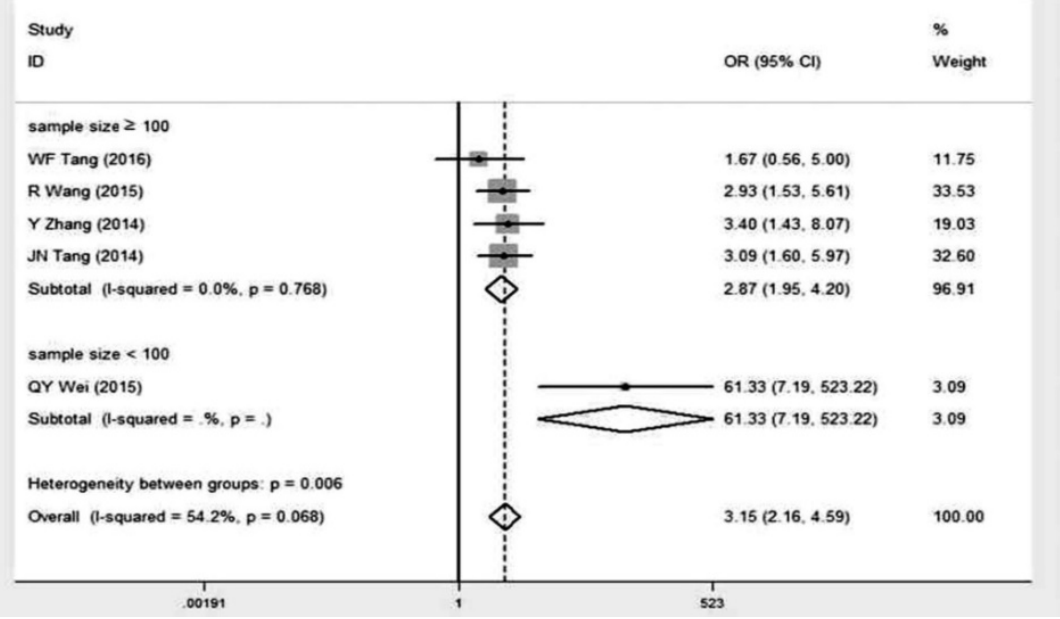

Figure 3. Meta-analysis of association between high GOLPH3 expression and clinical stage of lung cancer. A. Odds ratio (OR) with $95 \% \mathrm{Cl}$ for the association between high GOLPH3 expression and clinical stage of patients, III-IV stage vs I-II stage. B. Subgroup analysis of OR based on sample size, III-IV stage vs I-II stage. If OR $>1$ and the $95 \%$ CI doesn't include 1, it indicates that there is an association between high GOLPH3 expression and advanced clinical stage of patients with lung cancer.

Study

ID
$\%$

OR $(95 \% \mathrm{Cl}) \quad$ Weight
WF Tang (2016)

R Wang (2015)

QY Wei (2015)

M Lu (2014)

Y Zhang (2014)

XL Lv (2014)

Overall (1-squared $=40.0 \%, p=0.139)$

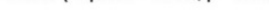

\begin{tabular}{|c|c|c|}
\hline Overall (1-squared $=40.0 \%, p=0.139$ ) & $1.97(1.34,2.88)$ & 100.00 \\
\hline${ }_{0328}^{1}$ & 30.7 & \\
\hline
\end{tabular}

Figure 4. Forest plot of odds ratio (OR) with $95 \% \mathrm{Cl}$ for the association between high GOLPH3 expression and tumor differentiation in NSCLC, moderate to poor differentiation vs well differentiation. If OR $>1$ and the $95 \% \mathrm{Cl}$ doesn't include 1, it indicates that there is an association between high GOLPH 3 expression and poor differentiation of tumor. 
A

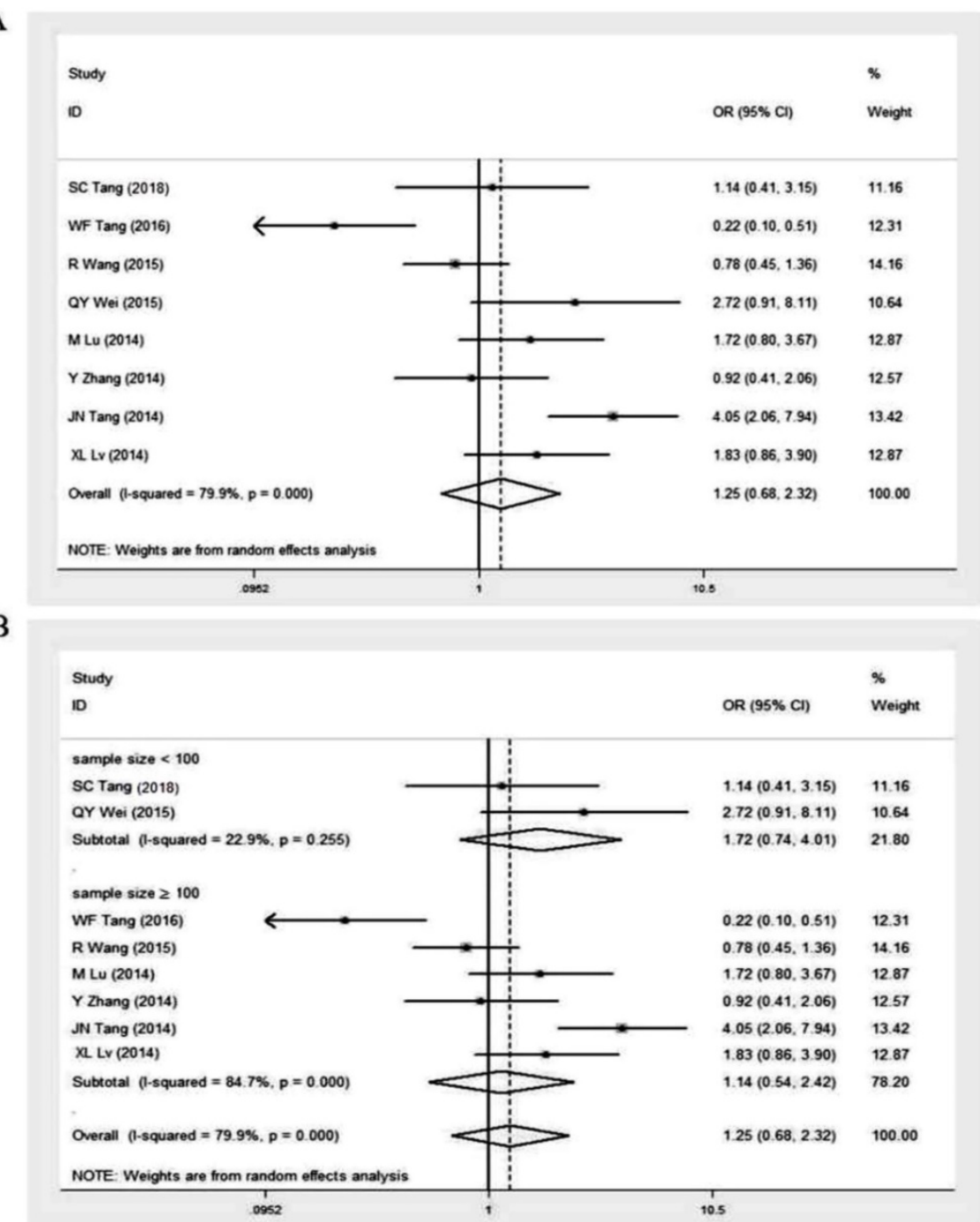

Figure 5. Meta-analysis of association between high GOLPH3 expression and histologic type of lung cancer. A. Odds ratio (OR) with $95 \% \mathrm{Cl}$ for the association between high GOLPH3 expression and histologic type of lung cancer, lung squamous cell carcinoma vs lung adenocarcinoma. B. Subgroup analysis of OR based on sample size, lung squamous cell carcinoma vs lung adenocarcinoma. If OR $>1$ and the $95 \% \mathrm{Cl}$ doesn't include 1, it indicates that there is an association between high GOLPH3 expression and histologic type of lung cancer.

Table 3. Odds ratios of GOLPH3 overexpression in lung cancer tissues according to gender, age and tumor size with subgroup analysis based on study sample size or cut-off.

\begin{tabular}{lllllll}
\hline Variable & Subgroup & OR & $95 \% \mathrm{CI}$ & $\mathrm{z}$-value & $\mathrm{p}$-value & $\mathrm{I}^{2}$ \\
\hline gender & overall & 1.19 & $(0.78-1.80)$ & 0.80 & 0.42 & $53.6 \%$ \\
& sample size $\geq 100$ & 1.22 & $(0.77-1.94)$ & 0.84 & 0.40 & $59.1 \%$ \\
& sample size $<100$ & 1.05 & $(0.26-4.26)$ & 0.07 & 0.94 & $64.0 \%$ \\
age & cut-off $=60$ years & 1.13 & $(0.67-1.91)$ & 0.44 & 0.66 & $24.5 \%$ \\
& cut-off $=65$ years & 0.96 & $(0.59-1.56)$ & 0.18 & 0.85 & $51.8 \%$ \\
tumor size & cut-off $=3 \mathrm{~cm}$ & $1.98(0.85-4.60)$ & 1.59 & 0.11 & $61.0 \%$ \\
& cut-off $=5 \mathrm{~cm}$ & $1.48(0.78-2.83)$ & 1.19 & 0.24 & $0.0 \%$ \\
& T descriptor $\left(\mathrm{T}_{2} / \mathrm{T}_{1}\right)$ & 1.47 & $(0.99-2.18)$ & 1.93 & 0.05 & $0.0 \%$ \\
\hline
\end{tabular}

\section{Sensitivity analysis and publication bias}

In order to examine the stability of the pooled HR in OS analysis, sensitivity analysis was conducted and the results showed that exclusion of individual studies did not modify the overall results (Figure 8).
We employed the Egger's test to detect publication bias. The results indicated that no evidence for publication bias was observed in this meta-analysis (Figure 9).

Table 4: Summary of $\mathrm{HR}$ with $95 \% \mathrm{Cl}$ for OS and PFS in each study

\begin{tabular}{llll}
\hline \multirow{2}{*}{ Author } & \multicolumn{2}{l}{ OS (HR with 95\% CI) } & \multicolumn{1}{l}{ PFS (HR with 95\% CI) } \\
\cline { 2 - 4 } & Univariate analysis & Multivariate analysis & Multivariate analysis \\
\hline SC Tang & $1.63(1.21-2.20) /$ Rep & $1.71(1.25-2.33) /$ Rep & - \\
WF Tang & $3.91(1.79-8.50) /$ Rep & $5.67(1.52-21.11) /$ Rep & - \\
R Wang & $1.23(0.72-2.11) /$ KM & - & - \\
QY Wei & $2.45(0.93-6.47) /$ KM & - & - \\
M Lu & $2.23(1.17-4.20) /$ KM & $1.90(1.02-3.53) /$ Rep & $1.83(1.03-3.25) /$ Rep \\
Y Zhang & - & - & $3.58(1.83-7.01) /$ Rep \\
JN Tang & $1.86(1.13-3.04) /$ Rep & $2.19(1.29-3.73) /$ Rep & - \\
X L Lv & $1.77(0.98-3.20) /$ KM & - & -
\end{tabular}

OS: Overall survival; PFS: Progression-free survival; HR: Hazard ratios; Rep: Reported in the included studies;

KM: Calculated from Kaplan-Meier curve. 
A

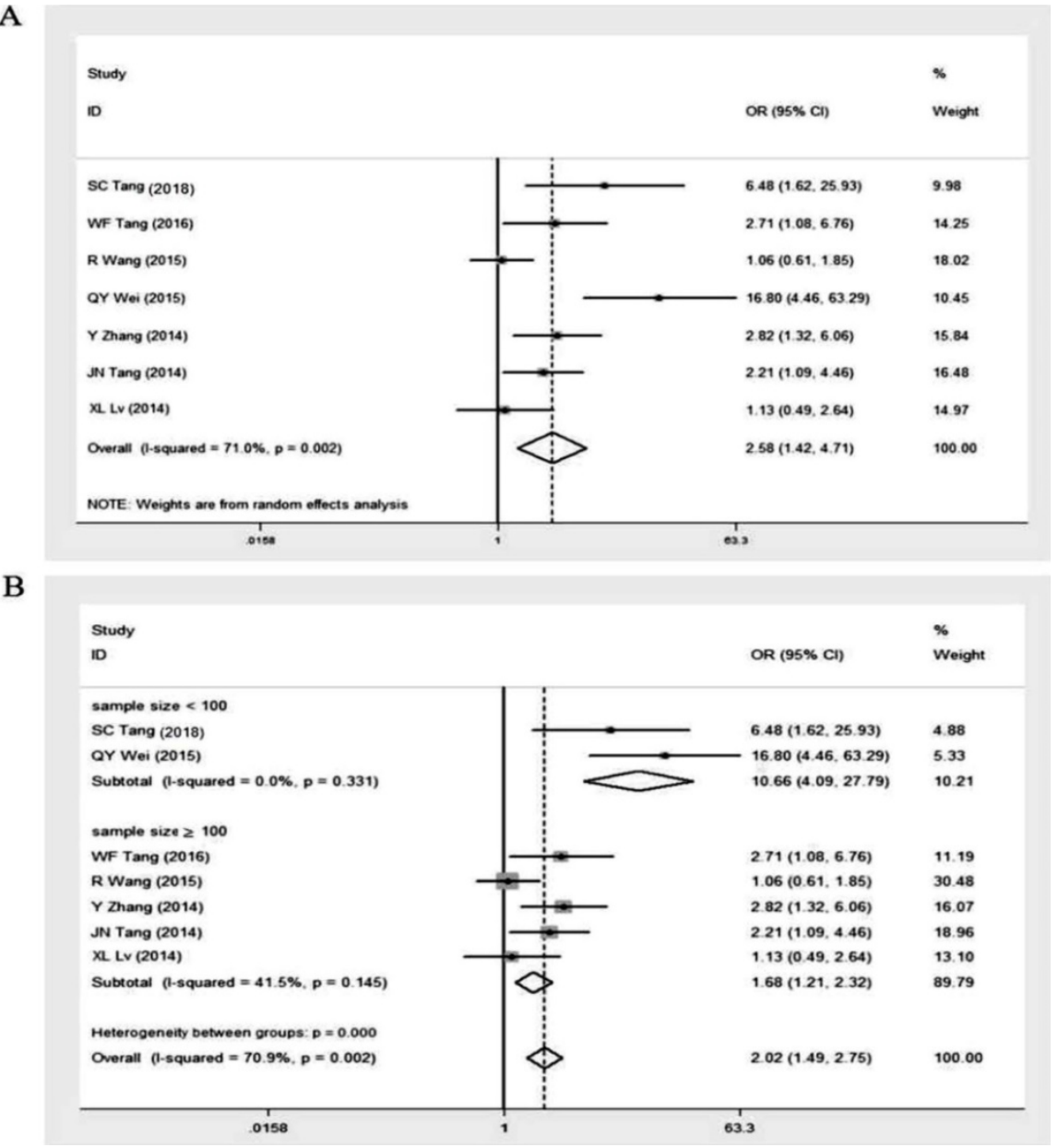

Figure 6. Meta-analysis of association between high GOLPH3 expression and lymphatic metastasis. A. Odds ratio (OR) with $95 \% \mathrm{Cl}$ for the association between high GOLPH3 expression and lymphatic metastasis, nodal metastasis vs no nodal metastasis. B. Subgroup analysis of OR based on sample size, nodal metastasis vs no nodal metastasis. If OR > 1 and the $95 \% \mathrm{Cl}$ doesn't include 1, it indicates that there is an association between high GOLPH3 expression and lymph node metastasis.

\section{Discussion}

Lung cancer has been a major public health problem and the number of new lung cancer cases is still on the rise largely as a result of an increase in global tobacco use, particularly in the developing countries [1]. However, only one third of patients are diagnosed at an early and potentially curable stage, and up to $50 \%$ of these patients will develop local or distant recurrences [30, 31]. Despite advances in surgical and nonsurgical therapy, the overall survival in cancer patients continues to be poor, with low 5 -year survival rates of $21 \%$ in women and $15 \%$ in men [32]. Increasing evidence suggested that the development of tumor biomarkers is crucial for early diagnosis, prognosis assessment and personalized therapy of cancer patients, including the patients with NSCLC. New strategies for identifying sensitive and reliable biomarker for accurate diagnosis and reliable prediction recurrences have attracted a great deal of interest in recent years.

GOLPH3 is a highly conserved protein located in chromosome 5p13, a region that is frequently amplified in multiple solid tumor types [33, 34]. It has been demonstrated that GOLPH3 moves between the trans-Golgi network and endosomal structures and serves a vital role in multiple cellular processes, such as trafficking, receptor recycling and protein glycosylation [35, 36]. As many proto-oncogenes encode glycosylated proteins involved in tumor cell proliferation, adhesion, invasion, migration, immune recognition and signal transduction [37], GOLPH3 may be associated with tumorigenesis and malignant biological behavior of cancer [35]. Further studies showed that GOLPH3 promotes the development of cancer by activating mammalian target of rapamycin (mTOR) signaling, enhancing AKT activity, and decreasing forkhead box O1 (FOXO1) transcriptional activity [13]. Recently, it has been reported that GOLPH3 overexpression was involved in cisplatin resistance and the detailed molecular mechanisms were associated with activation of the mitogen-activated protein kinase/extracellular regulated kinase and $W n t / \beta$-catenin signaling pathways [38]. Knockdown GOLPH3 could reduce cell proliferation and induce apoptosis, leading to 
tumor growth restriction and increased sensitivity of tumor cells to cisplatin [38, 39]. Therefore, it is speculated that GOLPH3 plays an essential role in tumor initiation and progression in some types of cancer.

In spite of our efforts to reach an accurate and comprehensive meta-analysis, the meta-analysis also has certain limitations. First, all included studies were retrospective and conducted in Asia with small sample sizes, which may result in potential selection bias. Second, the cut-off values for high/positive expression of GOLPH3 in the eligible studies were different, this inconsistency could have played a role in achieving the outcomes mentioned in this article. Third, several survival data were extracted from the Kaplan-Meier curves according to the method of Tierney et al [19], which could have impact on the credibility of the prognostic effect. Finally, statistical heterogeneity existed among studies. However, random effects model for these analyses and subgroup analysis were conducted to minimize such an impact.
In conclusion, our study demonstrated that GOLPH3 overexpression was positively correlated with adverse clinicopathologic features of NSCLC patients, with a tendency for poor prognosis, suggesting that GOLPH3 plays a vital role in the progression of NSCLC and may serve as a potential prognostic biomarker for NSCLC patients.

\section{Abbreviations}

NSCLC: non-small-cell lung carcinoma; GOLPH3: Golgi phosphoprotein 3; HR: hazard ratio; OR: odds ratio; IHC: immunohistochemistry; OS: overall survival; PFS: progression-free survival; mTOR: mammalian target of rapamycin; FOXO1: forkhead box protein $\mathrm{O} 1$.

\section{Acknowledgement}

We thank Dr. Yang Song in Pfizer medical for providing helpful suggestions. This study was supported by the National Natural Science Foundation of China (Grant No. 81670051).
$\mathbf{A}$

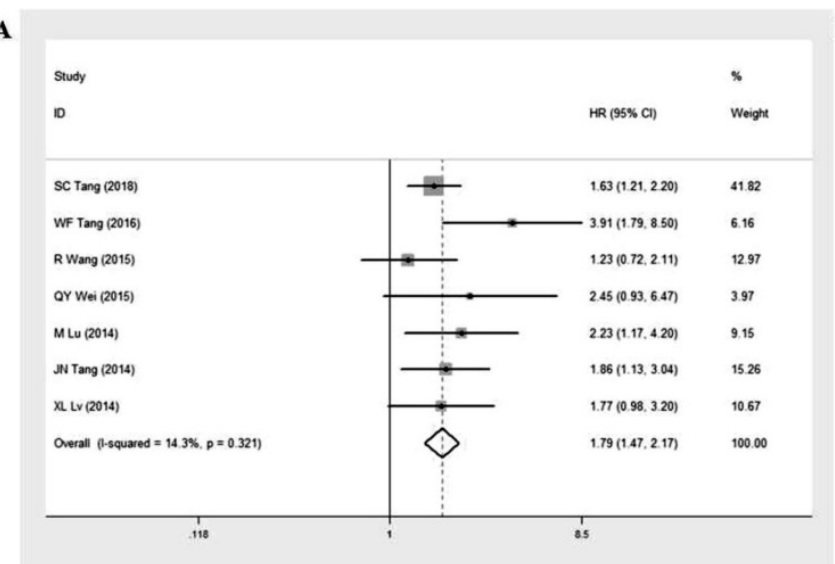

C

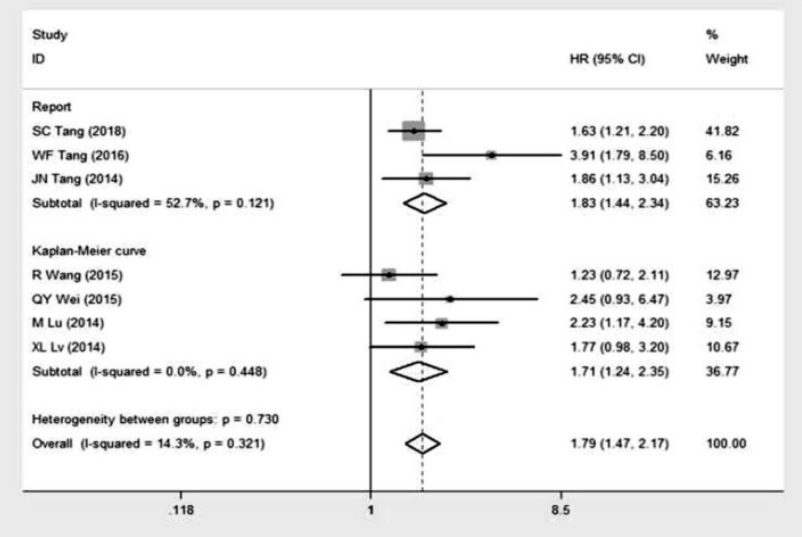

B

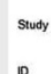

ID

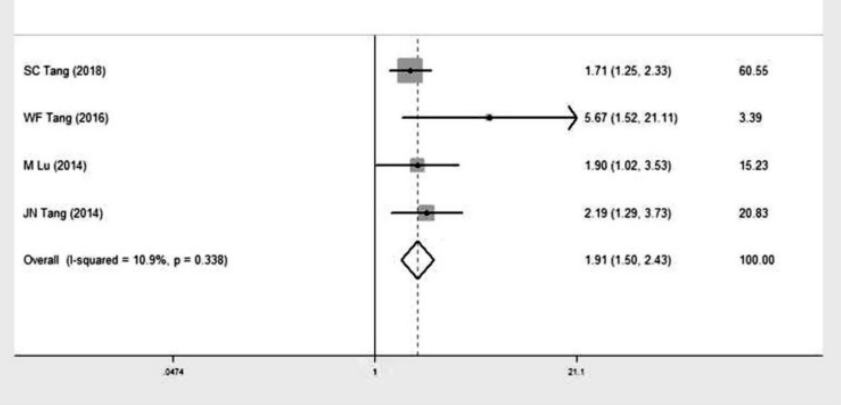

D

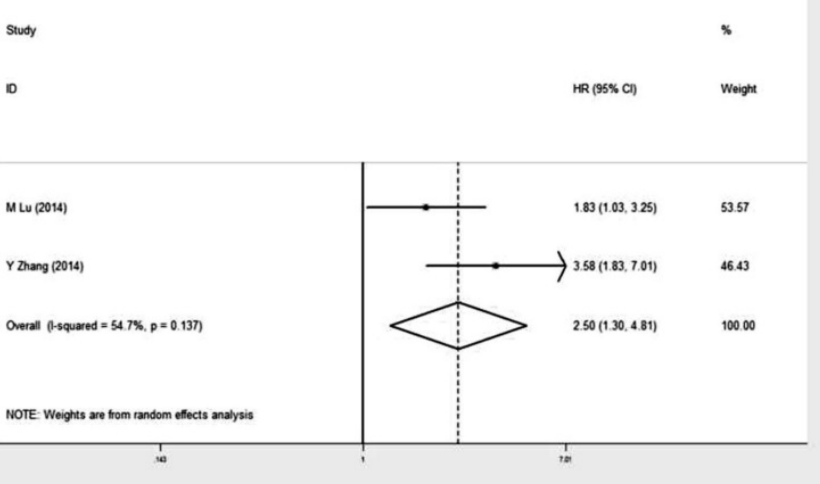

Figure 7. Forest plots for the prognostic meta-analysis. A. Hazard ratio (HR) with $95 \% \mathrm{Cl}$ for the association between high GOLPH3 expression and overall survival (OS) in univariate analysis, high vs low GOLPH3 expression. B. HR with $95 \% \mathrm{Cl}$ for the association between high GOLPH3 expression and OS in multivariate analysis, high vs low GOLPH3 expression. C. Subgroup analysis of HR based on the method of obtaining HRs with $95 \% \mathrm{Cl}$ for OS in univariate analysis, high vs low GOLPH3 expression. D. HR with $95 \% \mathrm{Cl}$ for the association between high GOLPH3 expression and progression free survival (PFS) in multivariate analysis, high vs low GOLPH3 expression. If HR > 1 and the $95 \%$ $\mathrm{Cl}$ doesn't include 1, it represents that patients with high GOLPH3 expression have a poor prognosis. 


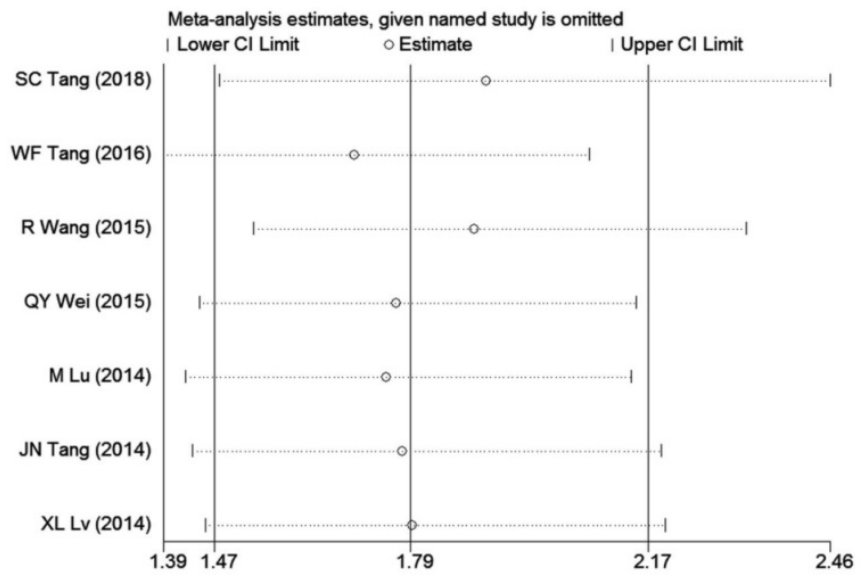

Figure 8. Forests plots of sensitivity analysis for the relationship between high GOLPH3 expression and overall survival (OS) in patients with lung cancer.

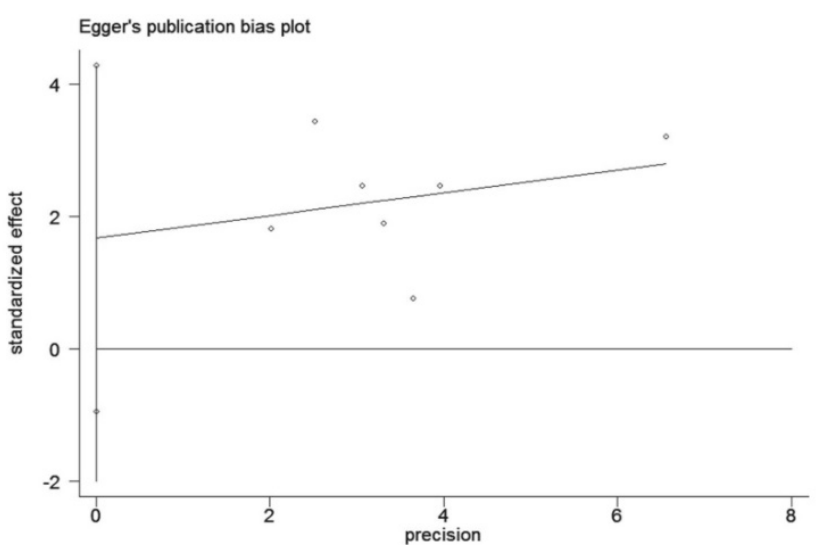

Figure 9. Egger's test for publication bias in GOLPH3 impact on the overall survival (OS) in patients with lung cancer.

\section{Competing Interests}

The authors have declared that no competing interest exists.

\section{References}

1. Siegel RL, Miller KD, Jemal A. Cancer statistics, 2018. CA Cancer J Clin. 2018;68(1):7-30

2. Parkin DM, Bray F, Ferlay J, et al. Global cancer statistics, 2002. CA Cancer J Clin. 2005;55(2):74-108.

3. Siegel R, Naishadham D, Jemal A. Cancer statistics, 2012. CA Cancer J Clin. 2012;62(1):10-29.

4. Reungwetwattana T, Dy GK. Targeted therapies in development for non-small cell lung cancer. J Carcinog.2013;12:22.

5. Petrella F, Spaggiari L. Therapeutic options following pneumonectomy in non-small cell lung cancer. Expert Rev Respir Med.2016;10(8):919-925.

6. Barzi A, Pennell NA. Targeting Angiogenesis in Non-Small Cell Lung Cancer: Agents in Practice and Clinical Development. European J Clin Med Oncol.2010; 2(1):31-41.

7. Leppert W, Zdun-Ryzewska A, Forycka M, et al. Quality of life assessment in advanced cancer patients treated at home, an inpatient unit, and a day care center. Onco Targets Ther.2014;7:687-695.

8. Wu CC, Taylor RS, Lane DR, et al. GMx33: a novel family of trans-Golgi proteins identified by proteomics. Traffic.2000;1(12):963-975

9. Dippold HC, Ng MM, Farber-Katz SE, et al. GOLPH3 bridges phosphatidylinositol-4- phosphate and actomyosin to stretch and shape the Golgi to promote budding. Cell.2009;139(2):337-51.

10. Zhou J, Xu T, Oin $\mathrm{R}$, et al. Overexpression of Golgi phosphoprotein-3 (GOLPH3) in glioblastoma multiforme is associated with worse prognosis. J Neurooncol. 2012;110(2):195-203.
11. Tang S, Yang R, Zhou X, et al. Expression of GOLPH3 in patients with non-small cell lung cancer and xenografts models. Oncol Lett.2018;15(5):7555-62.

12. Peng J, Fang Y, Tao Y, et al. Mechanisms of GOLPH3 associated with the progression of gastric cancer: a preliminary study. PLoS One.2014;9(10):e107362.

13. Zeng $\mathrm{Z}$, Lin $\mathrm{H}$, Zhao $\mathrm{X}$, et al. Overexpression of GOLPH3 promotes proliferation and tumorigenicity in breast cancer via suppression of the FOXO1 transcription factor. Clin Cancer Res.2012;18(15):4059-4069.

14. Dai $\mathrm{T}$, Zhang D, Cai M, et al. Golgi phosphoprotein 3 (GOLPH3) promotes hepatocellular carcinoma cell aggressiveness by activating the NF-kappaB pathway. J Pathol.2015;235(3):490-501.

15. Hua X, Yu L, Pan W, et al. Increased expression of Golgi phosphoprotein-3 is associated with tumor aggressiveness and poor prognosis of prostate cancer. Diagn Pathol.2012;7:127.

16. Tang W, Han M, Ruan B, et al. Overexpression of GOLPH3 is associated with poor survival in Non-small-cell lung cancer. Am J Transl Res.2015;8(4):1756-1762.

17. Wang MZ, Qiu CZ, Yu WS, et al. GOLPH3 expression promotes the resistance of HT29 cells to 5fluorouracil by activating multiple signaling pathways. Mol Med Rep.2018;17(1):542-548.

18. Parmar MK, Torri V, Stewart L. Extracting summary statistics to perform meta-analyses of the published literature for survival endpoints. Stat Med.1998; 17(24):2815-2834

19. Tierney JF, Stewart LA, Ghersi D, et al. Practical methods for incorporating summary time-to-event data into meta-analysis. Trials.2007;8:16.

20. Stang A. Critical evaluation of the Newcastle-Ottawa scale for the assessment of the quality of nonrandomized studies in meta-analyses. Eur J Epidemiol. 2010;25(9):603-605

21. Higgins JP, Thompson SG, Deeks JJ, et al. Measuring inconsistency in meta-analyses. BMJ.2003;327(7414):557-560.

22. Higgins JP. Commentary: Heterogeneity in meta-analysis should be expected and appropriately quantified. Int J Epidemiol.2008;37(5):1158-1160.

23. Egger M, Davey SG, Schneider M, et al. Bias in meta-analysis detected by a simple, graphical test. BMJ. 1997;315(7109):629-634.

24. Wang R, Ke ZF, Wang F, et al. GOLPH3 overexpression is closely correlated with poor prognosis in human non-small cell lung cancer and mediates its metastasis through upregulating MMP-2 and MMP-9. Cell Physiol Biochem.2015;35(3):969-982.

25. Zhang Y, Ma M, Han B. GOLPH3 high expression predicts poor prognosis in patients with resected non-small cell lung cancer: an immunohistochemical analysis. Tumour Biol.2014;35(11):10833-10839.

26. Lu M, Tian Y, Yue WM, et al. GOLPH3, a good prognostic indicator in early-stage NSCLC related to tumor angiogenesis. Asian Pac J Cancer Prev 2014;15(14):5793-5798.

27. Wei QY. The relationships of GOLPH3 expression with tumor cell apoptosis and clinicaopathological features in NSCLC [D]. Fujian medical university. 2015.

28. Tang JN, Wen XN, Bai J. Expression of GOLPH3 in NSCLC and Its Prognostic Significance. J Med Res.2015(2):126-130.

29. Lv XL, Tian H, Lu M, et al. Golgi phosphoprotein 3 expression in non-small cell lung cancer and its clinical significance. Chin J Cancer Biother.2014; 21(5): 554-558.

30. Novello S, Le Chevalier T. Chemotherapy for non-small-cell lung cancer. Part 1: Early-stage disease. Oncology (Williston Park).2003;17(3):357-364.

31. Visbal AL, Leighl NB, Feld R, et al. Adjuvant Chemotherapy for Early-Stage Non-small Cell Lung Cancer. Chest.2005;128(4):2933-2943.

32. Chalian H, Khoshpouri P, Assari S. Demographic, Social, and Behavioral Determinants of Lung Cancer Perceived Risk and Worries in a National Sample of American Adults; Does Lung Cancer Risk Matter? Medicina (Kaunas).2018;54(6): 97.

33. Gorringe KL, Boussioutas A, Bowtell DD. Novel regions of chromosomal amplification at $6 \mathrm{p} 21,5 \mathrm{p} 13$, and $12 \mathrm{q} 14$ in gastric cancer identified by array comparative genomic hybridization. Genes Chromosomes Cancer.2005;42(3):247-259.

34. Bohm M, Wieland I, Schmidt C, et al. Loss of heterozygosity on chromosome 5 p13-12 predicts adverse prognosis in advanced bladder cancer independent of tumor stage and grade. J Urol.2002;168(6):2655-2658.

35. Ohtsubo K, Marth JD. Glycosylation in cellular mechanisms of health and disease. Cell.2006;126(5):855-867.

36. Dippold HC, Ng MM, Farber-Katz SE, et al. GOLPH3 bridges phosphatidylinositol-4- phosphate and actomyosin to stretch and shape the Golgi to promote budding. Cell.2009;139(2):337-351.

37. Takahashi M, Tsuda $\mathrm{T}$, Ikeda $\mathrm{Y}$, et al. Role of $\mathrm{N}$-glycans in growth factor signaling. Glycoconj J.2004;20(3):207-212.

38. Zhou ZP, Wang LP, Hong ZS, et al. Silencing GOLPH3 gene expression reverses resistance to cisplatin in HT29 colon cancer cells via multiple signaling pathways. Int J Oncol.2018;53(3):1183-1192.

39. Liu H, Wang X, Feng B, et al. Golgi phosphoprotein 3 (GOLPH3) promotes hepatocellular carcinoma progression by activating mTOR signaling pathway. BMC Cancer.2018:18(1):661. 